\title{
A MEMS Accelerometer for Sub-mG Sensing
}

\author{
Daisuke Yamane, ${ }^{1 *}$ Toshifumi Konishi, ${ }^{2}$ Teruaki Safu, ${ }^{2}$ Hiroshi Toshiyoshi, ${ }^{3}$ \\ Masato Sone, ${ }^{1}$ Katsuyuki Machida, ${ }^{1}$ Hiroyuki Ito, ${ }^{1}$ and Kazuya Masu ${ }^{1}$ \\ ${ }^{1}$ Tokyo Institute of Technology, \\ 4259 Nagatsuta-cho, Midori-ku, Yokohama, Kanagawa 226-8503, Japan \\ ${ }^{2}$ NTT Advanced Technology Corporation, \\ 3-1 Wakamiya, Morinosato, Atsugi, Kanagawa 243-0124, Japan \\ ${ }^{3}$ The University of Tokyo, \\ 4-6-1 Komaba, Meguro-ku, Tokyo 153-8904, Japan
}

(Received August 29, 2018; accepted December 10, 2018)

Keywords: MEMS, accelerometer, thermomechanical noise, Au, multilayer metal technology

In this paper, we present a highly sensitive micro-electromechanical system (MEMS) accelerometer for sub-mG sensing resolution, where the thermomechanical noise (i.e., Brownian noise, $B_{N}$ ) being inversely proportional to a proof mass has to be below $1 \mu G / \sqrt{\mathrm{Hz}}$, (gravity acceleration $G=9.8 \mathrm{~m} / \mathrm{s}^{2}$ ). To increase the proof mass, we propose the use of multiple-layered metal developed by Au electroplating. We then show an approach to design the spring constant for the MEMS accelerometer. A multilayer metal structure is used for serpentine flexures to suspend the high-density proof mass, which also enables us to obtain a high degree of freedom for the spring constant design without compromising the performance of the MEMS accelerometer. A proof-of-concept device has been fabricated, and the measured characteristics are consistent with the design values. The $B_{N}$ of the developed device is experimentally evaluated to be $22 \mathrm{n} G / \sqrt{\mathrm{Hz}}$, which is one or more orders of magnitude lower than those of conventional MEMS accelerometers with the same capacitance sensitivity. The evaluation results confirm that the proposed device has potential for sub-mG sensing.

\section{Introduction}

Micro-electromechanical system (MEMS) accelerometers have become key devices for inertial sensing technology in a variety of applications. ${ }^{(1,2)}$ The sensing resolution of MEMS accelerometers for commercial use is commonly designed to sense more than $1 \mathrm{mG}$ (gravity acceleration $\left.G=9.8 \mathrm{~m} / \mathrm{s}^{2}\right)$, ${ }^{(3,4)}$ and the resolution has to be further improved for future potential applications such as space applications, seismometry, and inertial navigation. ${ }^{(5,6)}$ To realize high-resolution acceleration sensing, it is necessary to reduce the noise in the MEMS accelerometer. Thermomechanical noise (i.e., Brownian noise, $B_{N}$ ) is one of the dominant noise sources in MEMS accelerometers. ${ }^{(7-9)}$ Conventionally, large proof masses made of silicon were used to reduce $B_{N}$, since $B_{N}$ is inversely proportional to the proof mass. ${ }^{(7-9)}$ Such methods need

*Corresponding author: e-mail: yamane.d.aa@m.titech.ac.jp

https://doi.org/10.18494/SAM.2019.2122 
bulk-micromachining and/or wafer bonding techniques ${ }^{(10-18)}$ and result in large sensor modules. Thus far, the $B_{N}$ 's of surface micromachined accelerometers are higher than $10 \mu G / \sqrt{\mathrm{Hz}},{ }^{(19-21)}$ and $B_{N}$ below $1 \mu G / \sqrt{\mathrm{Hz}}$ is only shown by bulk micromachined accelerometers for micro-G resolution..$^{(8,9,14,15)}$ In our previous works, we have proposed high-resolution MEMS accelerometers with a high-density Au proof mass ${ }^{(22,23)}$ developed by Au electroplating. ${ }^{(24)}$

In this paper, we present a Au proof-mass MEMS accelerometer for sub-mG sensing. Multilayer metal structures are newly employed for both the proof mass and the spring in the MEMS accelerometer, which contribute to the decrease in $B_{N}$. Analysis and experimental results confirm the feasibility of the proposed approach. In the following sections, we first show a design concept of the device and design methods for the proof mass and spring structures. Next, a device fabrication process using multilayer metal technology is described. Finally, we show the experimental evaluation results and the performance comparison with conventional devices.

\section{Device Design and Fabrication}

In this section, we describe the design concept of the proposed MEMS accelerometer and materials used. Then, design methods for the proof mass and spring structures are shown with analysis results. Lastly, we present the device fabrication process.

\subsection{Design concept}

Figure 1 shows the design concept of a single-axis capacitive ${ }^{(19-21)}$ MEMS accelerometer with a $\mathrm{Au}$ proof mass. For a high-resolution capacitive MEMS accelerometer, both the mechanical and electrical noises have to be minimized. The major part of mechanical noise is dominated by $B_{N}$, which is given by

$$
B_{N}=\frac{\sqrt{4 k_{B} T b}}{m}
$$

where $k_{B}, T, b$, and $m$ are the Boltzmann constant $\left(1.38 \times 10^{-23} \mathrm{~J} / \mathrm{K}\right)$, absolute temperature, viscous damping coefficient, and proof mass of an accelerometer, respectively. ${ }^{(19)}$ As shown in Eq. (1), $B_{N}$ is inversely proportional to the proof mass. MEMS capacitive accelerometers conventionally utilize Si mechanical structures and employ a large Si proof mass when a low $B_{N}$ is required. We previously proposed an alternative approach ${ }^{(22,23)}$ to reducing $B_{N}$ in a MEMS accelerometer, where we used a $\mathrm{Au}$ proof mass developed by a multilayer metal technology. ${ }^{(24)}$ The density of $\mathrm{Au}\left(19.3 \times 10^{3} \mathrm{~kg} / \mathrm{m}^{3} \text { at } 298 \mathrm{~K}\right)^{(25)}$ is nearly an order of magnitude higher than that of $\mathrm{Si}\left(2.33 \times 10^{3} \mathrm{~kg} / \mathrm{m}^{3}\right.$ at $\left.298 \mathrm{~K}\right),{ }^{(25)}$ and thus we can achieve $B_{N}$ lower than that of $\mathrm{Si}$ proof mass of the same dimensions.

For sub-mG $\left(<10^{-3} G\right)$ sensing, the target $B_{N}$ of this work was determined to be below $50 \mathrm{n} G / \sqrt{\mathrm{Hz}},{ }^{(23)}$ where the frequency band is set to be $10 \mathrm{~Hz} .{ }^{(23)}$ The total noise of a capacitive accelerometer consists of $B_{N}$ and electrical noise. ${ }^{(26)}$ In this study, we assume that the electrical noise is negligibly small, which can be achieved by considering the sensing circuit technology ${ }^{(27)}$ and large sensitivity, typically on the order of several $\mathrm{pF} / G$. 
Figure 2 shows the analysis results of $B_{N}$ as a function of proof-mass size, where $T$ and $b$ are set to be $298 \mathrm{~K}$ and $1.85 \times 10^{-5} \mathrm{~N} \cdot \mathrm{s} / \mathrm{m}$, respectively. The thickness of the Si proof mass is set to be $10 \mu \mathrm{m}$, which is a typical value of conventional surface-micromachined MEMS accelerometers. ${ }^{(28-30)}$ Owing to its high density, the Au proof mass has a lower $B_{N}$ than a $\mathrm{Si}$ proof mass. Thus, we have developed Au proof mass structures with a thickness of more than $12 \mu \mathrm{m}$. ${ }^{(23)}$ To achieve $B_{N}$ below $50 \mathrm{n} G \sqrt{\mathrm{Hz}}$, the thickness of the Au proof mass has to be increased. The proof mass of a MEMS accelerometer developed by the multilayer metal technology has release holes for sacrificial etching; thus, a 4-mm-square proof mass with a thickness of $10 \mu \mathrm{m}$ is insufficient to achieve a noise level below $50 \mathrm{n} G \sqrt{\mathrm{Hz}}$. In this work, we designed a multilayer Au proof mass with a total thickness of $20 \mu \mathrm{m}$. As the thickness of a single $\mathrm{Au}$ layer in the multilayer technology was smaller than $20 \mu \mathrm{m}$, we proposed to utilize multiple Au layers for the proof mass. The proof-mass structure of $20 \mu \mathrm{m}$ thickness can be achieved by using two Au layers in the multilayer metal technology. In this design, we assume that the proof mass is a perforated plate with equally spaced release holes, and the viscous damping coefficient $b$ can be expressed by ${ }^{(31)}$

$$
\begin{gathered}
b=0.427 N \mu \frac{L^{4}}{d^{3}}, \\
L=\sqrt{p^{2}-a^{2}},
\end{gathered}
$$

where $N, \mu, d$, and $L$ are the number of damping holes, the viscosity of the surrounding gas, the air gap, and the effective proof-mass length with the release hole pitch $p$ and size $a$, respectively. We use $1.8 \times 10^{-5} \mathrm{~N} \cdot \mathrm{s} / \mathrm{m}^{2}$ for the viscosity of air ${ }^{(32)}$, and $N, d, p$, and $a$ are designed to be 15376 , $5.92 \mu \mathrm{m}, 16 \mu \mathrm{m}$, and $8.7 \mu \mathrm{m}$, respectively. As a result, $b$ is estimated to be $1.85 \times 10^{-5} \mathrm{~N} \cdot \mathrm{s} / \mathrm{m}$.

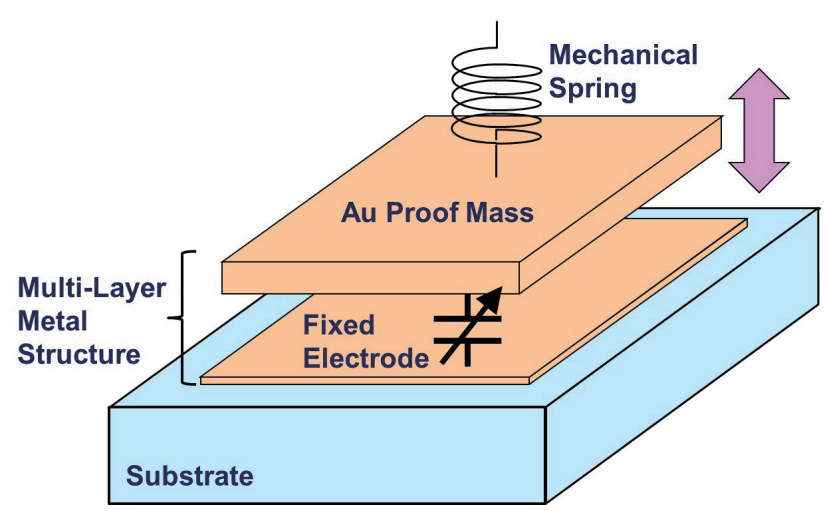

Fig. 1. (Color online) Schematic of a high-density Au proof-mass MEMS capacitive accelerometer.

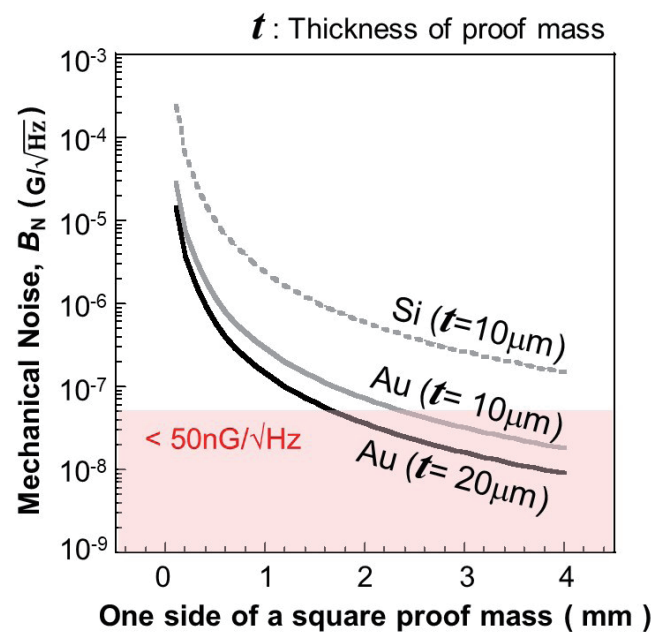

Fig. 2. (Color online) Mechanical noise analysis results. 


\subsection{Spring structure}

The Au proof mass can become one order of magnitude higher than the Si proof mass as shown in Fig. 3; thus, the spring constant of micromechanical flexure has to be larger than those of conventional Si types. Figure 4(a) shows an analytical model of the offset displacement of the proof mass at the input of the gravitational acceleration of $1 \mathrm{G}$, and the analysis results of the offset displacement $d$ of a 4-mm-square proof mass are shown in Fig. 4(b). In a practical manner, the offset displacement of the Au proof mass has to be designed to be as small as that of a silicon proof mass. The analysis results indicate that the spring constant used for the Au proof mass should be nearly one order of magnitude higher than that of silicon MEMS accelerometers.

Serpentine spring structures ${ }^{(33)}$ would be useful for multiaxis acceleration sensing with a single proof mass, because the flexibility of tuning a 3-axis spring constant is higher than that of simple structures such as cantilever springs. Figure 5(a) shows an analytical model of a serpentine spring structure. Calculated $Z$-axis spring constants of serpentine flexures made of $\mathrm{Au}$ are shown in Fig. 5(b). Table 1 shows the analysis formula of the serpentine flexure, where we assume that the model is a guided-end spring with a concentrated load as the end and that $L_{c}$ is equal to $L_{a}{ }^{(33)}$ In Fig. 5(b), we used typical dimensions of the MEMS accelerometer fabricated by the multilayer metal technology; ${ }^{(34)}$ the parameters $L_{a}, L_{b}$, and $w$ were set to be 200, 10, and $6 \mu \mathrm{m}$, respectively. Young's modulus and Poisson's ratio of Au used in the analysis were $78 \mathrm{GPa}$ and 0.44 , respectively. ${ }^{(35)}$ The spring constants of $3-\mu \mathrm{m}$-thick serpentine flexures are insufficient to suspend a Au proof mass with a practical offset displacement up to $1 \mu \mathrm{m}$, as shown in Fig. 4(b). For a higher stiffness in the $Z$-axis direction, we designed a serpentine flexure of $15 \mu \mathrm{m}$ thickness and proposed to use the third and fourth Au layers in the multilayer metal technology. Using multiple Au layers, which have been newly introduced in this work, enables us to increase the degree of freedom of MEMS design in the multilayer metal technology. Reducing flexure lengths, such as $L_{a}$ and $L_{b}$, results in a shorter tuning range for stiffness. For instance, in order to achieve the $Z$-axis spring constant of a 15 - $\mu$ m-thick serpentine flexure, the $L_{a}$ of a 3- $\mu \mathrm{m}$-thick serpentine flexure has to be smaller than $w$, and such design cannot be allowed.

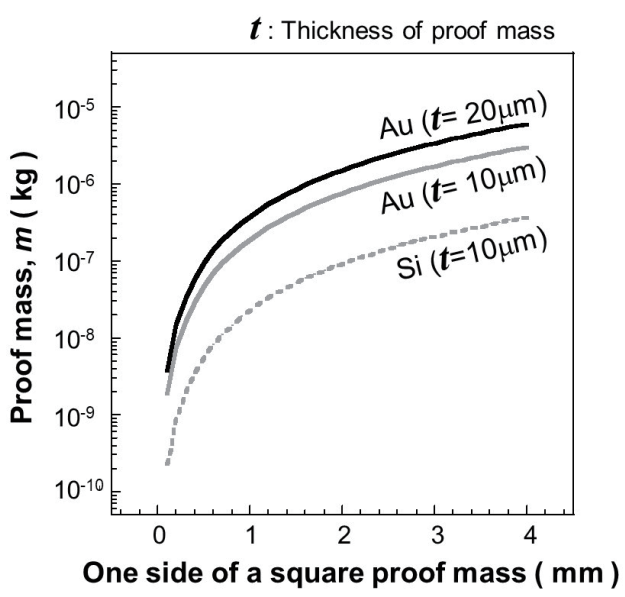

Fig. 3. Proof mass analysis results. 


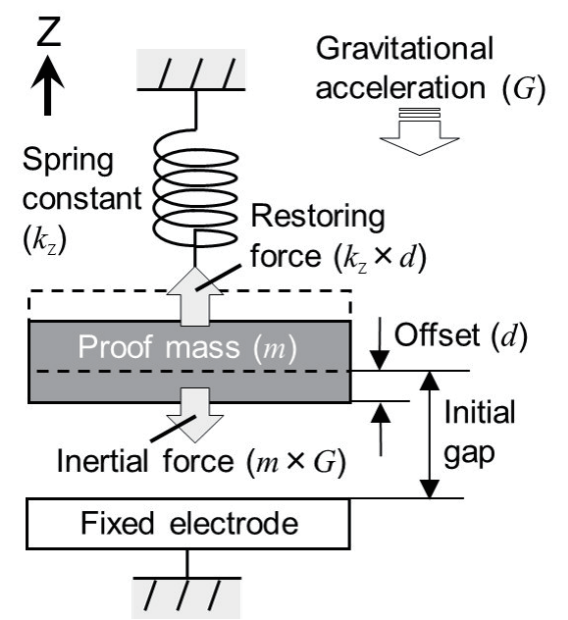

(a)

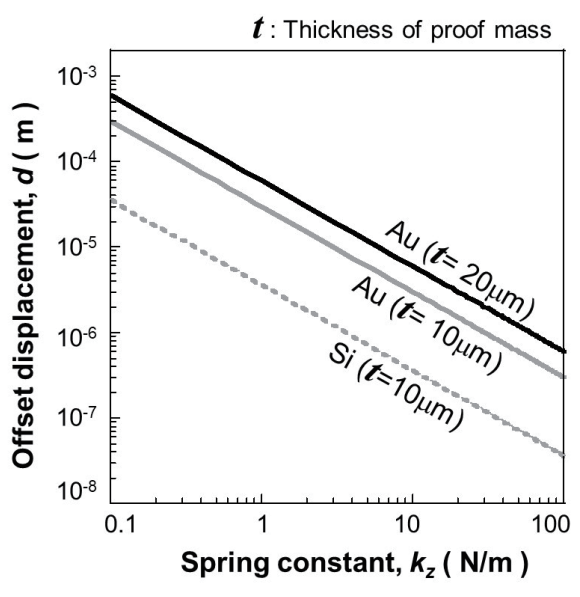

(b)

Fig. 4. Proof-mass offset with different spring constant. (a) Analytical model and (b) analysis results.

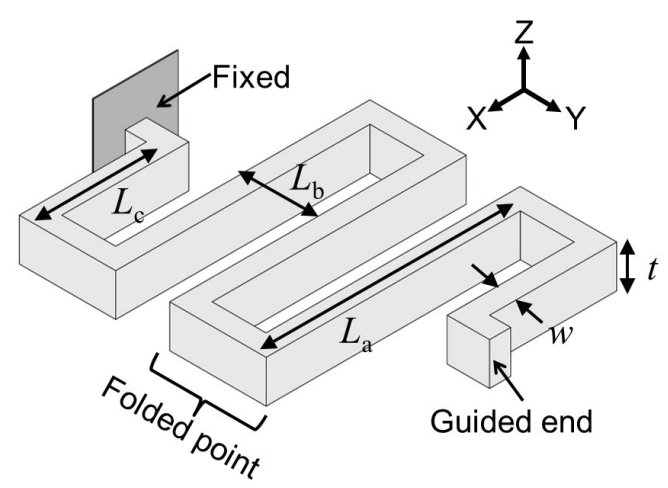

(a)

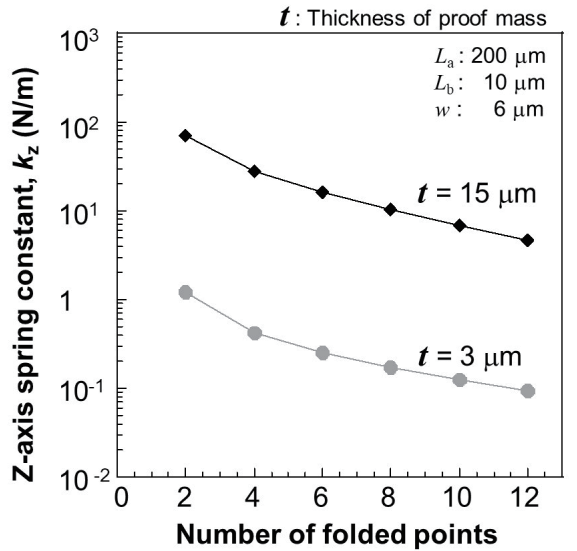

(b)

Fig. 5. Spring constant of serpentine structure. (a) Analytical model and (b) analysis results.

Table 1

Analysis formula of serpentine flexure.

\begin{tabular}{|c|c|}
\hline Axis & Spring constant of serpentine flexure \\
\hline Z & 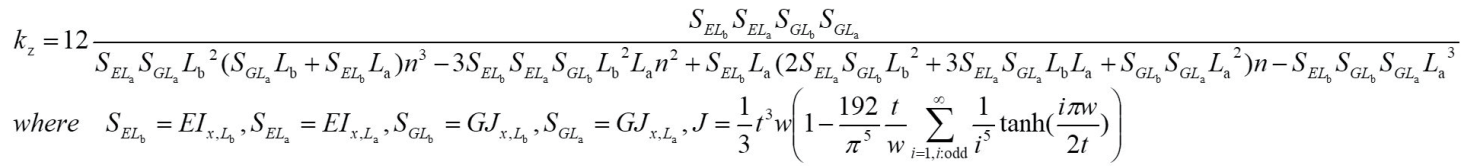 \\
\hline
\end{tabular}




\subsection{Device fabrication}

Figure 6 shows a schematic image of the proposed single-axis MEMS capacitive accelerometer. The device is realized by the multilayer technology based on electroplating, which has been reported elsewhere. ${ }^{(36)}$ As mentioned in the device design methods, we utilize the third (M3) and fourth (M4) layers for the spring structure, and the M4 and fifth (M5) layers for the proof mass. The stoppers are immobilized structures and used to prevent the self-destruction of the movable parts at the input of excessive acceleration. When an excess acceleration is applied to the device, the proof mass collides with either the stopper or the bottom fixed electrode, protecting the spring structures from stretching out of the elastic deformation range. Figure 7 shows the device fabrication process flow. Firstly, Ti/Au seed layers were deposited by evaporation on a silicon wafer with a thermally formed $\mathrm{SiO}_{2}$ layer, as shown in Fig. 7(a). A Au electroplating process was then used to increase the thickness of the first Au layer (M1). For sacrificial layer fabrication, photosensitive polyimide was spincoated and annealed at a temperature of $310{ }^{\circ} \mathrm{C}$. A SiO 2 layer of $1 \mu \mathrm{m}$ thickness was deposited by sputtering [Fig. 7(b)]. With the same Au patterning as for the M1 layer, we made another five Au layers (M2-M6), as illustrated in Fig. 7(c). Finally, all the sacrificial layers made of photosensitive polyimide were removed by $\mathrm{O}_{2}$ plasma dry etching [Fig. 7 (d)].

Figure 8(a) shows a chip view of the developed MEMS accelerometer. A Au proof mass was fabricated on a silicon substrate with a footprint of $4 \times 4 \mathrm{~mm}^{2}$. The device was implemented in a ceramic package and wire-bonded to have electrical connections with external instruments for experimental evaluation. The SEM image of the proof mass is shown in Fig. 8(b). The Au proof-mass structure of $22 \mu \mathrm{m}$ thickness was successfully developed by employing the M4 and M5 layers. To minimize the effect of the metal warpage, the proof mass was segmented into sub-blocks that were cross-linked by the M6 layer. Through-holes were made to enhance the

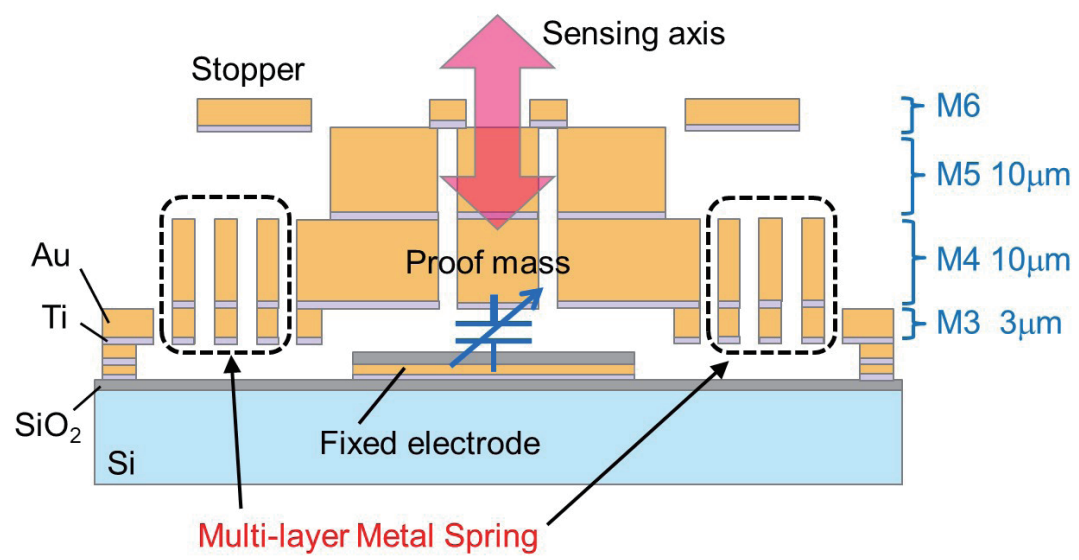

Fig. 6. (Color online) Schematic cross section of multilayer metal spring structures in a Au proof-mass MEMS capacitive accelerometer. 


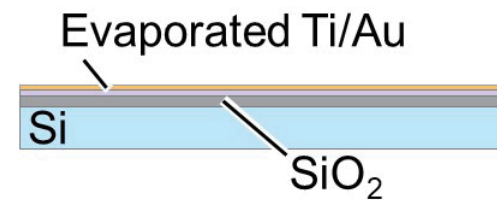

(a)

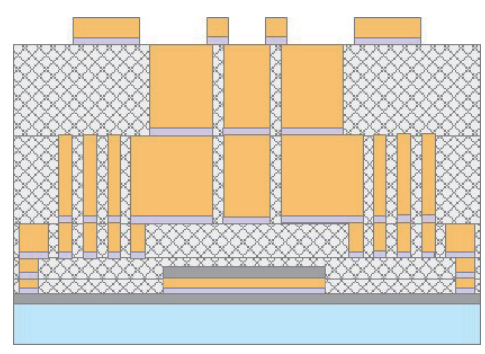

(c)

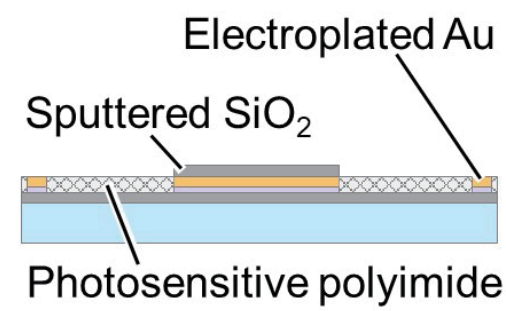

(b)

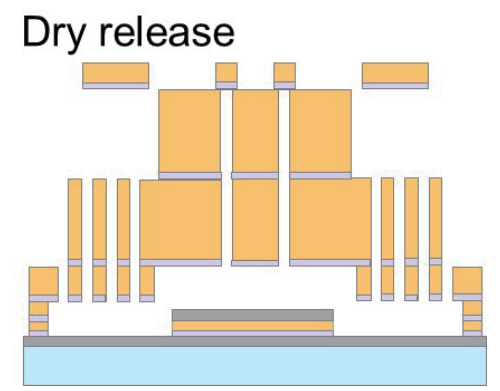

(d)

Fig. 7. (Color online) Process flow. (a) Seed layer deposition, (b) $\mathrm{M} 1$ and $\mathrm{SiO}_{2}$ patterning, (c) $\mathrm{M} 2-\mathrm{M} 6$ patterning, and (d) sacrificial layer etching.

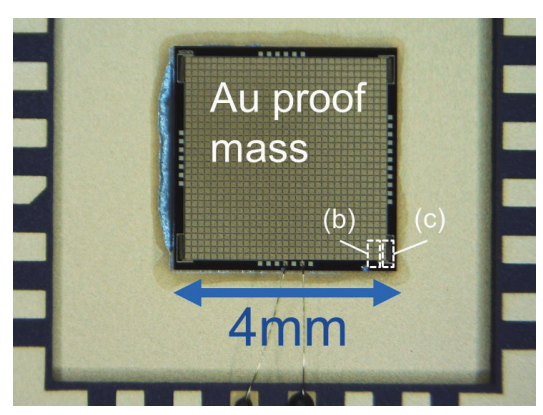

(a)

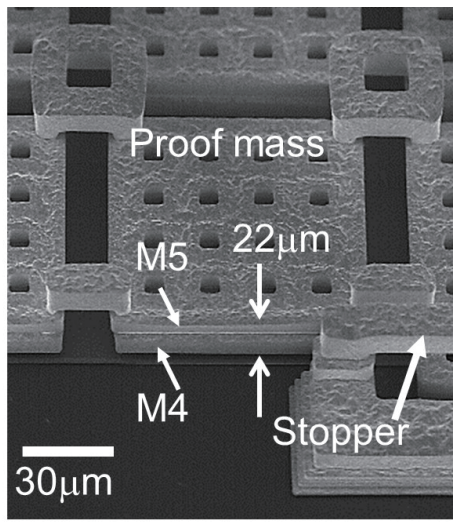

(b)

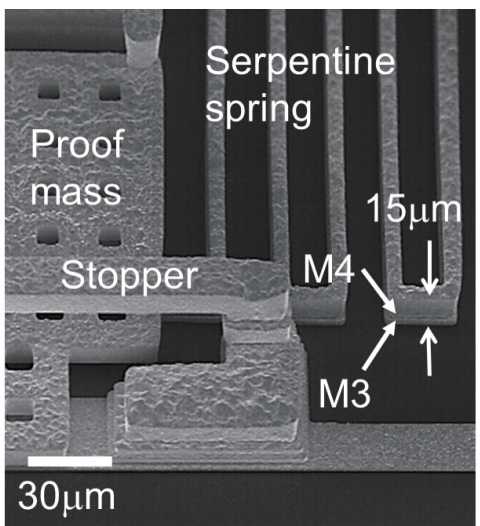

(c)

Fig. 8. (Color online) Fabricated device. (a) Chip view, (b) proof mass, and (c) spring structure. 
sacrificial layer etching underneath the proof mass. Figure 8(c) shows the SEM image of the serpentine spring structure made of M3 and M4 layers. Stopper structures were made of the M6 layer and set above the proof mass. The serpentine springs and stoppers were placed at each corner of the proof mass.

\section{Experimental Results and Discussion}

\subsection{Acceleration responses}

We measured the capacitance between the proof mass and the fixed electrode when Z-axis acceleration was applied to the device as shown in Fig. 9. Figure 9(a) shows the experimental setup, where the packaged MEMS device was set on a custom-designed printed circuit board (PCB) on a vibration exciter (WaveMaker05, Asahi Seisakusho) with a minimum input-acceleration step of $0.01 \mathrm{G}$. A semiconductor device analyzer (B1500A, Agilent Tech., Inc.) was used to supply a DC bias voltage of $0.5 \mathrm{~V}$ and measure the capacitance change with a $\pm 0.1 \mathrm{~V}$ sinusoidal voltage at a frequency of $300 \mathrm{kHz}$. Figure 9(b) shows the measured capacitance as a function of input acceleration at a frequency of $49.9 \mathrm{~Hz}$. The sensitivity was experimentally obtained to be $3.3 \mathrm{pF} / \mathrm{G}$, which showed the potential of sub-mG sensing with capacitance-to-voltage converter circuits. ${ }^{(27)}$

\subsection{Frequency characteristics}

For the evaluation of mechanical characteristics and $B_{N}$, we measured the frequency responses of the fabricated MEMS device. Figure 10 shows the capacitance and phase between the proof mass and the fixed electrode as a function of signal frequency. Figure 10(a) shows the experimental setup with an LCR meter (IM3533-01, HIOKI E.E. Corp.). As shown in Fig. 10(b), the resonant frequency of the device was found to be $202 \mathrm{~Hz}$ with a DC bias voltage of 0.5 V. The actual proof-mass size was measured by an SEM. The difference between the designed

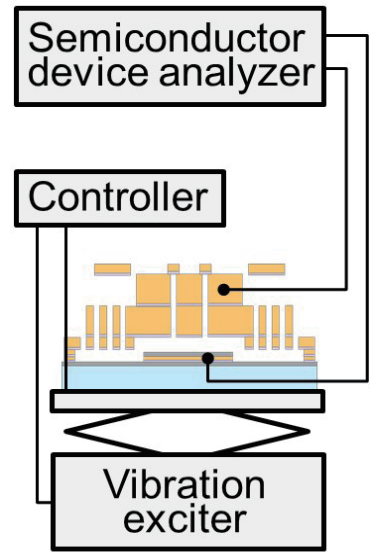

(a)

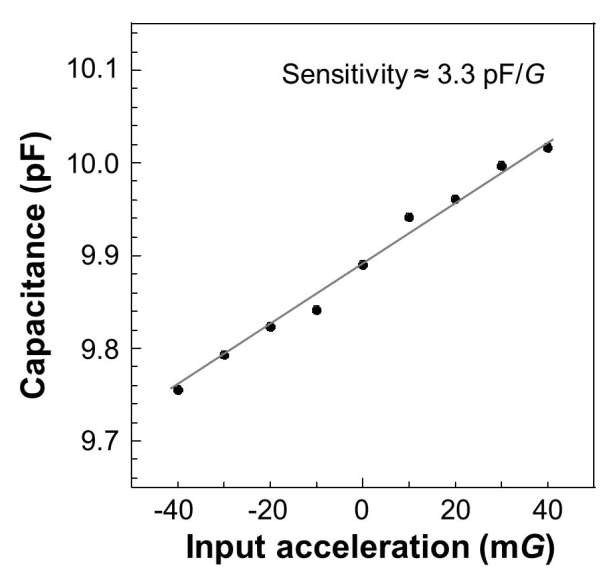

(b)

Fig. 9. (Color online) Measured capacitance as a function of input acceleration. (a) Experimental setup and (b) measurement results. 


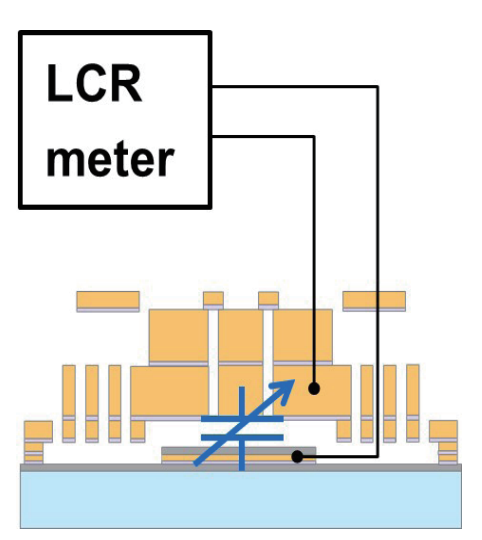

(a)
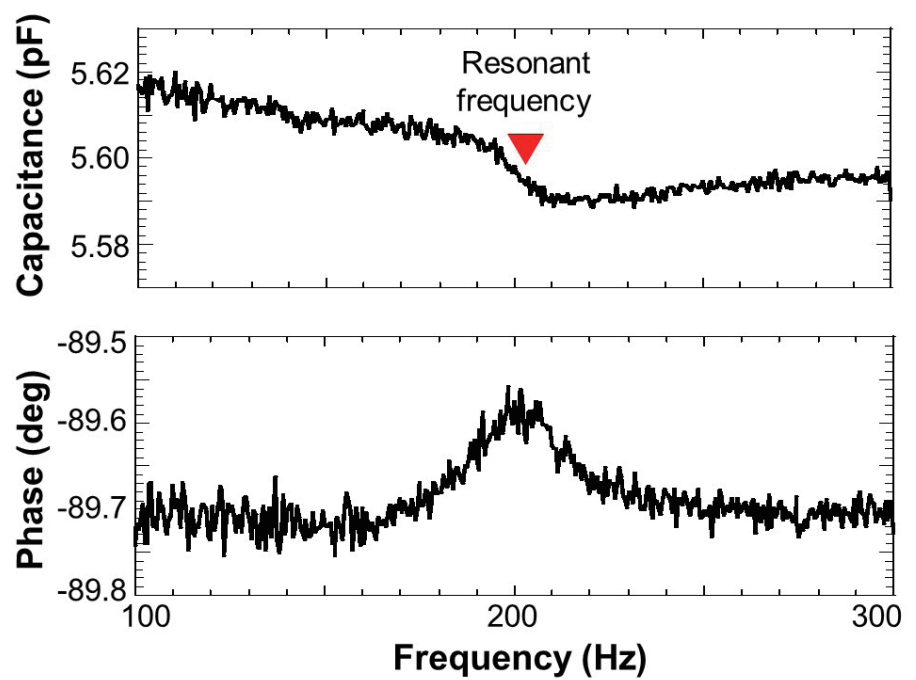

(b)

Fig. 10. (Color online) Measured capacitance and phase as a function of frequency. (a) Experimental setup and (b) measurement results.

and measured spring constants could be made by process variation that affects the dimension of serpentine spring structures. To evaluate the $B_{N}$ of the device, the following relationship ${ }^{(23)}$ was used:

$$
B_{N}=\sqrt{\frac{4 k_{B} T \omega_{r}}{m Q}}
$$

where $\omega_{r}$ and $Q$ are the resonant angular frequency and quality factor, respectively. The designed $Q$ is expressed as ${ }^{(32)}$

$$
Q=\frac{m \omega_{r e s}}{b},
$$

where $\omega_{\text {res }}$ is the resonant angular frequency. In the device design, the viscous damping coefficient $b$ of $1.85 \times 10^{-5} \mathrm{~N} \cdot \mathrm{s} / \mathrm{m}$ is used, as mentioned in Sect. 2.1. Table 2 shows a summary of the designed and measured characteristics of the device, and the actual $B_{N}$ was obtained to be $22 \mathrm{nG} / \sqrt{\mathrm{Hz}}$. The measured resonant frequency, spring constant, and quality factor are slightly lower than the designed values. The inconsistency could be caused by the difference between the designed and actual structure dimensions, and also by the warpage of the metal structures. Further investigation of these factors will be performed in our future works. Figure 11 shows a comparison of $B_{N}$ versus capacitance sensitivity with conventional MEMS accelerometers. Owing to the high density of $\mathrm{Au}$, the $B_{N}$ achieved in this work was more than an order of magnitude lower than those of conventional devices when compared with the same sensitivity performance. 
Table 2

Summary of device characteristics.

\begin{tabular}{lccc}
\hline Parameter & Designed value & Measured value & Unit \\
\hline Proof mass & $3.28 \times 10^{-6}$ & $3.62 \times 10^{-6}$ & $\mathrm{~kg}$ \\
Z-axis resonant frequency & 260 & 202 & $\mathrm{~Hz}$ \\
Z-axis spring constant & 8.7 & 5.8 & $\mathrm{~N} / \mathrm{m}$ \\
Quality factor & 289 & 131 & $\mathrm{nG} / \sqrt{\mathrm{Hz}}$ \\
BN & 17 & 22 & \\
\hline
\end{tabular}

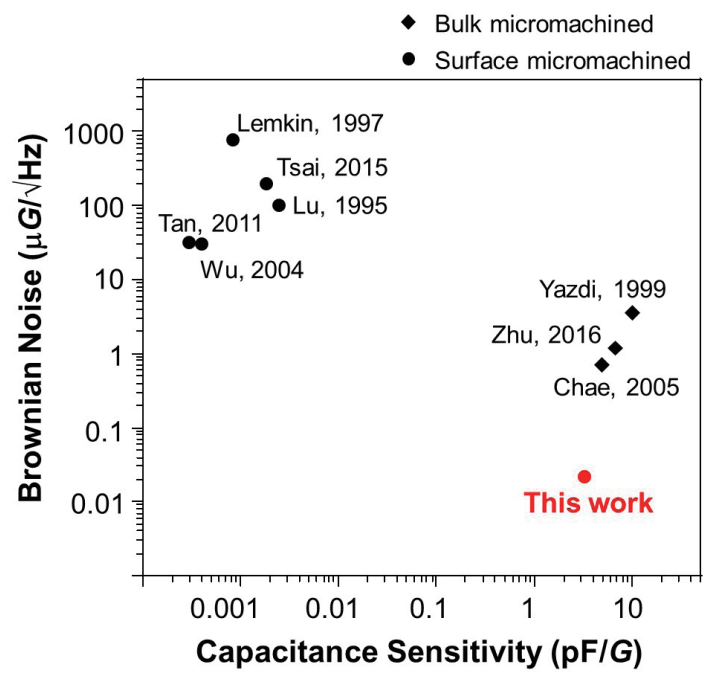

Fig. 11. (Color online) Comparison of BN versus capacitance sensitivity. ${ }^{(10,11,19-21,37-39)}$

\section{Conclusions}

We proposed and demonstrated a Au proof-mass MEMS accelerometer for sub-mG sensing. In our design method, multilayer metal structures were used for both proof mass and spring for the first time. The multilayer structures contribute to the decrease in $B_{N}$ without compromising the sensor performance and footprint. The measured characteristics of the developed MEMS devices were consistent with the designed values, demonstrating the feasibility of our approach. The experimentally obtained $B_{N}$ was found to be $22 \mathrm{nG} / \sqrt{\mathrm{Hz}}$. The $B_{N}$ of the developed sensor was more than one order of magnitude lower than those of conventional MEMS accelerometers with the same capacitance sensitivity. The evaluation results confirmed that the proposed MEMS device has potential for sub-mG sensing.

\section{Acknowledgments}

This work was supported by JST CREST Grant Number JPMJCR1433, Japan, and JSPS KAKENHI Grant Number 15K17453. 


\section{References}

1 D. K. Shaeffer: IEEE Commun. Mag. 51 (2013) 100. https://doi.org/10.1109/MCOM.2013.6495768

2 J. Laine and D. Mougenot: Proc. 2007 Int. Solid-State Sensors, Actuators and Microsystems Conf. (2007). https://doi.org/10.1109/SENSOR.2007.4300423

3 N. Yazdi, F. Ayazi, and K. Najafi: Proc. IEEE 86 (1998) 1640. https://doi.org/10.1109/5.704269

4 C. Acar and A. M. Shkel: J. Micromech. Microeng. 13 (2003) 634. https://doi.org/10.1088/0960-1317/13/5/315

5 G. Krishnan, C. U. Kshirsagar, G. K. Ananthasuresh, and N. Bhat: J. Indian Inst. Sci. 87 (2007) 333.

6 S. Scudero, A. D’Alessandro, L. Greco, and G. Vitale: Proc. 2018 IEEE Int. Conf. Environmental Engineering (2018). https://doi.org/10.1109/EE1.2018.8385252

7 M. Lemkin and B. E. Boser: IEEE J. Solid-State Circuits 34 (1999) 456. https://doi.org/10.1109/4.753678

8 B. V. Amini and F. Ayazi: J. Micromech. Microeng. 15 (2005) 2113. https://doi.org/10.1088/0960-1317/15/11/017

9 H. Kulah, J. Chae, N. Yazdi, and K. Najafi: IEEE J. Solid-State Circuits 41 (2006) 352. https://doi.org/10.1109/ JSSC.2005.863148

10 N. Yazdi, K. Najafi, and A. S. Salian: J. Microelectromech. Syst. 12 (2003) 479. https://doi.org/10.1109/ JMEMS.2003.815837

11 K.-H. Han and Y.-H. Cho: J. Microelectromech. Syst. 12 (2003) 11. https://doi.org/10.1109/ JMEMS.2002.805043

12 J. Chae, H. Kulah, and K. Najafi: J. Microelectromech. Syst. 14 (2005) 235. https://doi.org/10.1109/ JMEMS.2004.839347

13 B. V. Amini, R. Abdolvand, and F. Ayazi: IEEE J. Solid-State Circuits 41 (2006) 2983. https://doi.org/10.1109/ JSSC.2006.884864

14 R. Abdolvand, B. V. Amini, and F. Ayazi: J. Microelectromech. Syst. 16 (2007) 1036. https://doi.org/10.1109/ JMEMS.2007.900879

15 H. Qu, D. Fang, and H. Xie: IEEE Sensors J. 8 (2008) 1511. https://doi.org/10.1109/JSEN.2008.923582

16 Y. Dong, P. Zwahlen, A.-M. Nguyen, F. Rudolf, and J.-M. Stauffer: Proc. IEEE/ION Position, Location and Navigation Symposium (2010) 32. https://doi.org/10.1109/PLANS.2010.5507135

17 B. Homeijer, D. Lazaroff, D. Milligan, R. Alley, J. Wu, M. Szepesi, B. Bicknell, Z. Zhang, R. G. Walmsley, and P. G. Hartwell: Proc. 2011 IEEE 24th Int. Conf. Micro Electro Mechanical Systems (2011) 585. https://doi. org/10.1109/MEMSYS.2011.5734492

18 W. Zhu, Y. Zhang, G. Meng, C. S. Wallace, and N. Yazdi: Proc. 2016 IEEE 29th Int. Conf. Micro Electro Mechanical Systems (2016) 926. https://doi.org/10.1109/MEMSYS.2016.7421783

19 J. Wu, G. K. Fedder, and L. R. Carley: IEEE J. Solid-State Circuits 39 (2004) 722. https://doi.org/10.1109/ JSSC. 2004.826329

20 S.-S. Tan, C.-Y. Liu, L.-K. Yeh, Y.-H. Chiu, M. S.-C. Lu, and K. Y. J. Hsu: IEEE Trans. Circuit Syst. I 58 (2011) 2661. https://doi.org/10.1109/TCSI.2011.2142990

21 M.-H. Tsai, Y.-C. Liu, K.-C. Liang, and W. Fang: J. Microelectromech. Syst. 24 (2015) 1916. https://doi. org/10.1109/JMEMS.2015.2452270

22 T. Konishi, D. Yamane, T. Matsushima, G. Motohashi, K. Kagaya, H. Ito, N. Ishihara, H. Toshiyoshi, K. Machida, and K. Masu: Jpn. J. Appl. Phys. 52 (2013) 06GL04. https://doi.org/10.7567/JJAP.52.06GL04

23 D. Yamane, T. Konishi, T. Matsushima, K. Machida, H. Toshiyoshi, and K. Masu: Appl. Phys. Lett. 104 (2014) 074102. https://doi.org/10.1063/1.4865377

24 K. Machida, S. Shigematsu, H. Morimura, Y. Tanabe, N. Sato, N. Shimoyama, T. Kumazaki, K. Kudou, M. Yano, and H. Kyuragi: IEEE Trans. Electron Devices 48 (2001) 2273. https://doi.org/10.1109/16.954466

25 D. R. Lide: CRC Handbook of Chemistry and Physics (CRC Press, Boca Raton, FL, 1994) 75th ed.

26 P. Monajemi and F. Ayazi: IEEE Sensors J. 6 (2006) 39. https://doi.org/ 10.1109/JSEN.2005.854134

27 A. Utz, C. Walk, N. Haas, T. Fedtschenko, A. Stanitzki, M. Mokhtari, M. Gortz, M. Kraft, and R. Kokozinski: J. Sens. Sens. Syst. 6 (2017) 285. https://doi.org/10.5194/jsss-6-285-2017

28 W. Yun, R. T. Howe, and P. R. Gray: Proc. Technical Digest IEEE Solid-State Sensor and Actuator Workshop (1992) 126. https://doi.org/10.1109/SOLSEN.1992.228309

29 J. H. Smith, S. Montague, J. J. Sniegowski, J. R. Murray, and P. J. McWhorter: Proc. Int. Electron Devices Meeting (1995) 609. https://doi.org/10.1109/IEDM.1995.499295

30 M.-H. Tsai, Y.-C. Liu, and W. Fang: J. Microelectromech. Syst. 21 (2012) 1329. https://doi.org/10.1109/ JMEMS.2012.2205904

31 N. Yazdi and K. Najafi: J. Microelectromech. Syst. 9 (2000) 544. https://doi.org/10.1109/84.896777

32 S. D. Senturia: Microsystem Design (Springer Science+Business Media, Inc., New York, 2001).

33 G. K. Fedder: Ph.D. thesis, Dept. of Engineering-Eelectrical Engineering and Computer Sciences, University of California at Berkeley, Berkeley, California (1994). 
34 D. Yamane, T. Konishi, T. Safu, H. Toshiyoshi, M. Sone, K. Masu, and K. Machida: Proc. 2016 IEEE 11th Annu. Int. Conf. Nano/Micro Engineered and Molecular Systems (NEMS) (2016). https://doi.org/10.1109/ NEMS.2016.7758281

35 G. M. Rebeiz: RF MEMS: Theory, Design, and Technology (John Wiley \& Sons, Inc., Hoboken, New Jersey, 2003)

36 D. Yamane, T. Konishi, H. Toshiyoshi, K. Masu, and K. Machida: Proc. 2016 IEEE Int. Symp. Inertial Sensors and Systems (2016). https://doi.org/10.1109/ISISS.2016.7435571

37 C. Lu, M. Lemkin, and B. E. Boser: IEEE J. Solid-State Circuits 30 (1995) 1367. https://doi. org/10.1109/4.482163

38 M. A. Lemkin, B. E. Boser, D. Auslander, and J. H. Smith: Proc. Int. Solid State Sensors and Actuators Conf. (Transducers '97) (1997) 1185. https://doi.org/10.1109/SENSOR.1997.635417

39 N. Yazdi and K. Najafi: Proc. 1999 IEEE Int. Solid-State Circuits Conf.. Digest of Technical Papers. ISSCC. First Edition (Cat. No.99CH36278) (1999) 132. https://doi.org/10.1109/ISSCC.1999.759161 